\title{
Propagation et répercussions des maladies à transmission vectorielle émergentes à l'échelle mondiale
}

\author{
Kulkarni $\mathrm{MA}^{1 *}$
}

\author{
Affiliation \\ ${ }^{1}$ École d'épidémiologie, de santé publique et de médecine préventive, Université d’Ottawa, Ottawa (Ontario) \\ *Correspondance : manisha.kulkarni@uottawa.ca
}

Citation proposée : Kulkarni MA. Propagation et répercussions des maladies à transmission vectorielle émergentes à l'échelle mondiale. Relevé des maladies transmissibles au Canada 2016;42:221-2. https://doi.org/10.14745/ccdr.v42i10a02f

\section{Contexte}

Dans le cadre de la mondialisation croissante, les maladies contagieuses nouvelles et émergentes deviennent une priorité de santé mondiale. Les maladies à transmission vectorielle (MTV), en particulier celles transmises par les moustiques, comprennent une partie importante d'événements récents relatifs à des maladies infectieuses émergentes. Les facteurs tels que le changement climatique, l'évolution des agents pathogènes, l'aggravation de la lutte contre les vecteurs ainsi que les changements sociodémographiques et environnementaux, dans le contexte de la croissance de la population et de l'urbanisation incontrôlée et souvent rapide, peuvent faciliter la propagation géographique des infections, notamment des maladies transmises par les moustiques.

\section{Objectif}

Décrire les récentes tendances et répercussions mondiales des MTV émergentes et examiner les facteurs qui ont contribué à leur émergence et à leur propagation.

\section{Narratif}

La soudaine émergence du virus Zika dans les Amériques l'année passée, à la suite de son expansion en Afrique et en Asie, met en évidence la tendance à la hausse de la propagation mondiale des maladies transmises par les moustiques. La propagation des virus transmis par les moustiques Aedes, notamment le virus de la dengue, le virus Chikungunya et le virus Zika, constitue une préoccupation récente. Ces infections représentent une menace particulière pour les populations urbaines, surtout pour les pays à revenu faible et intermédiaire qui enregistrent des taux d'urbanisation rapides. Les fortes densités de population et le manque de logement et d'assainissement adéquats favorisent la reproduction des espèces de moustique Aedes aegypti et Aedes albopictus dans les conteneurs et les débris que l'on trouve habituellement près des maisons.

Le virus de la dengue, le virus Chikungunya et le virus Zika partagent les mêmes vecteurs et ont des origines écologiques similaires; on ne prévoit pas de cocirculation de ces virus dans de nombreuses parties du monde. Dans les zones endémiques de l'Afrique et de l'Asie, les virus restent à des cycles sylvatiques où ils circulent entre les hôtes réservoirs de la faune et les vecteurs moustiques. La transmission aux populations humaines a habituellement lieu lorsque des personnes empiètent sur les cycles sylvatiques, ce qui peut mener à la circulation du virus dans les populations de moustiques urbaines et, en fin de compte, à des éclosions majeures de la maladie (1). Les éclosions de maladies urbaines chez les populations naïves sur le plan immunitaire entraînent de forts taux de morbidité et de mortalité, en particulier dans les zones où les ressources de surveillance et de contrôle des vecteurs sont limitées. Le déplacement des personnes et des marchandises dans le cadre des déplacements et du commerce à l'échelle internationale peut entraîner la propagation des virus transmis par les moustiques dans les régions du monde où il existe des vecteurs compétents. Cette tendance est encore exacerbée par le changement climatique, qui augmente la possibilité de transmission des maladies à l'échelle locale dans de nombreuses régions qui n'étaient pas endémiques auparavant.

La propagation mondiale du virus Zika montre les facteurs clés qui peuvent entraîner l'émergence et l'expansion de MTV à l'échelle mondiale. Repéré pour la première fois en Ouganda en 1947, il a été signalé que le virus Zika causait une maladie bénigne sporadique en Afrique équatoriale et dans des parties de l'Asie (une éclosion a eu lieu en Micronésie en 2007). Par la suite, le virus s'est propagé dans la région du Pacifique, causant une importante éclosion en Polynésie française en 2013. II a ensuite été repéré pour la première fois dans les Amériques en 2014 (2). Depuis son émergence au Brésil, les premiers cas étant signalés au début de l'année 2015, le virus Zika s'est propagé dans pas moins de 35 pays des Amériques, causant un nombre inconnu d'infections et des milliers de cas de microcéphalie et de syndrome de Guillain-Barré contractés à l'échelle locale. Des cas d'infections transmissibles sexuellement sont désormais consignés dans des pays sans transmission locale. La propagation du virus Zika est attribuée au déplacement de voyageurs virémiques du Brésil vers des régions ayant des populations de moustiques Ae. aegypti établies (3). De plus, les températures exceptionnellement élevées et les conditions de sécheresse importantes dans le nord et l'est de l'Amérique 
du Sud, associées au récent courant El Niño pourraient avoir contribué à l'émergence rapide du virus Zika en 2015 (4). De plus, des changements moléculaires ont été remarqués dans la souche asiatique du virus Zika; ceux-ci pourraient être associés à la hausse de la pathogénicité et de la transmissibilité observée (5).

\section{Conclusion}

L'émergence et la propagation des MTV à l'échelle mondiale sont dues aux récents changements mondiaux. En plus de l'intervention en santé publique accrue, une meilleure compréhension de l'épidémiologie des MTV est nécessaire pour déterminer les facteurs de ces épidémies et éclairer I'intervention en santé publique. Ces éclosions sont des sources de nouveaux agents pathogènes qui pourraient arriver au Canada et ailleurs. Étant donné l'importance des MTV pour la santé mondiale et la vulnérabilité du Canada en ce qui concerne l'émergence des MTV, il faut renforcer de façon importante et urgente la capacité mondiale en matière de recherche, de surveillance, de prévention et d'intervention pour ce qui est des MTV. II est important de noter que l'émergence et l'expansion des maladies dans les pays limités en ressources ayant une faible capacité de surveillance et des systèmes de santé faibles font augmenter le risque d'introduction de la maladie dans d'autres pays en raison de l'interconnectivité mondiale croissante. Cela souligne la nécessité d'une coordination et de partenariats de santé à l'échelle mondiale pour lutter contre les MTV au Canada et à l'étranger.

\section{Conflit d'intérêts}

Aucun.

\section{Références}

1. Weaver SC. Urbanization and geographic expansion of zoonotic arboviral diseases: Mechanisms and potential strategies for prevention. Trends Microbiol 2013;21:360-3.

2. Kindhauser MK, Allen T, Frank V, Santhana RS, Dye C. Zika: the origin and spread of a mosquito-borne virus. Bull World Health Organ 2016;1-18.

3. Bogoch II, Brady OJ, Kraemer MU, German M, Creatore MI, Kulkarni MA et al. Anticipating the international spread of Zika virus from Brazil. Lancet 2016;387:335-6.

4. Paz S, Semenza JC. El Niño and climate changecontributing factors in the dispersal of Zika virus in the Americas? Lancet 2016;387:745.

5. Wang L, Valderramos SG, Wu A, Ouyang S, Li C, Brasil P et al. From Mosquitos to Humans: Genetic Evolution of Zika Virus. Cell Host Microbe 2016;19(5):561-5.

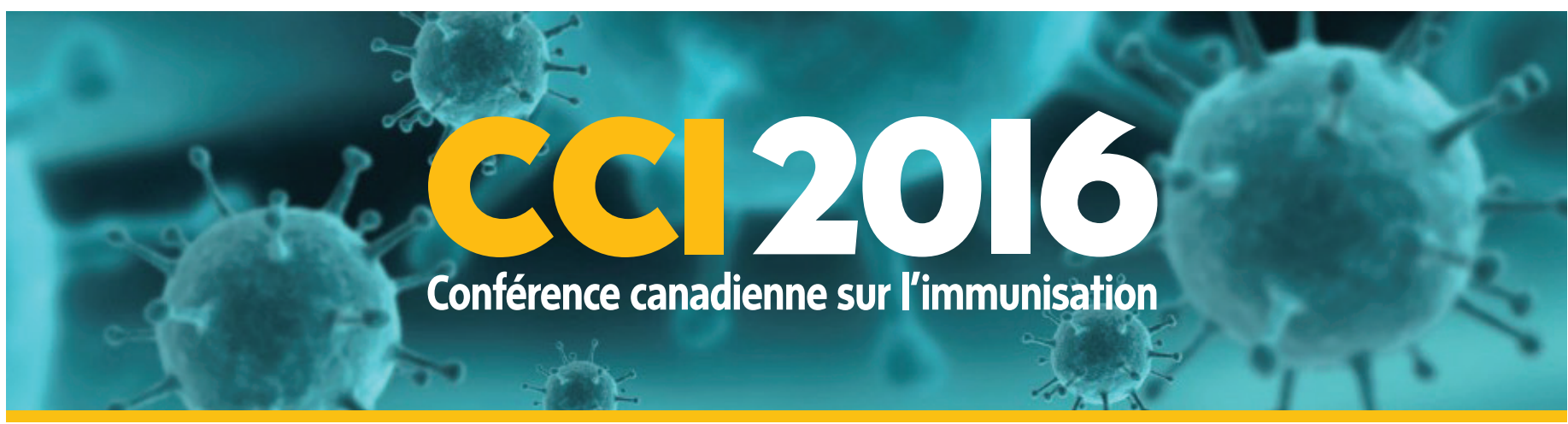

\title{
Conférence canadienne sur l'ïmmunisation 2016
}

\author{
Du 6 au 8 décembre 2016 | Centre Shaw \\ Ottawa (Ontario) Canada
}

\section{Le tarif d'inscription hâtive est en vigueur jusqu'au 11 octobre 2016}

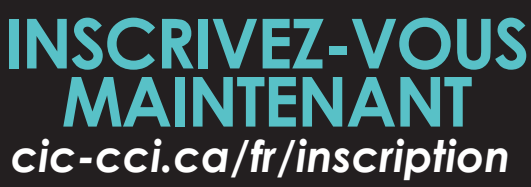
cic-cci.ca/fr/inscription 